\title{
ALTERAÇÕES COGNITIVAS NA INFECÇÃO PELO HIV E AIDS
}

\author{
Paulo Pereira Christo
}

Trabalho realizado no Núcleo de Ensino e Pesquisa da Santa Casa de Belo Horizonte. Hospital Eduardo de Menezes - FHEMIG, Belo Horizonte, MG

*Correspondência: Av. Professor Alfredo Balena, 189 / 1708 Santa Efigênia Belo Horizonte - MG CEP: $30130-100$

\section{RESUMO}

Dentre as complicações neurológicas primária da Aids temos déficits cognitivos como a demência associada ao HIV e formas mais leves, como o transtorno cognitivo/motor menor, sendo que ambas podem alterar as atividades da vida diária e reduzir a qualidade de vida dos pacientes. Infecção pelo HIV-1 é a mais comum, previsível e tratável causa de déficits cognitivos em indivíduos com menos de 50 anos. A despeito do avanço no conhecimento das características clínicas, patogênese, aspectos neurobiológicos e ao amplo uso de terapia antirretroviral altamente ativa (HAART), complicações neurológicas e déficits cognitivos ainda persistem levando a graves consequências pessoais e socioeconômicas tornando-se um grande desafio terapêutico. Na era pré- HAART, a demência era uma complicação comum da infecção, entretanto na era HAART a incidência da demência diminuiu, mas a prevalência tem aumentado principalmente das formas mais leves devido ao aumento do número de pessoas infectadas e ao aumento da expectativa de vida. Alterações cognitivas associadas ao HIV são tipicamente subcorticais e podem estar associadas a comprometimentos comportamentais e motores. Estas síndromes são de diagnóstico clínico, sendo que testes neuropsicológicos, neuroimagem e líquido cerebrorraquidiano corroboram no diagnóstico. Esta revisão faz uma atualização do estado atual da epidemiologia, características clínicas e diagnóstico das complicações cognitivas no curso da infecção pelo HIV.

UnItermos: Complexo aids demência. HIV. Síndrome de imunodeficiência adquirida. Cognição. Revisão.

\section{INTRODUÇÃO}

Desde seu reconhecimento, no início dos anos 80, a Síndrome da Imunodeficiência Adquirida (sida-aids) se disseminou pelo mundo tornando-se um dos maiores desafios de saúde pública das três últimas décadas.

A Organização Mundial de Saúde (OMS) estima que, no mundo, aproximadamente 33,2 milhões de pessoas estão infectadas pelo vírus HIV ou apresentam a doença e que em 2007 ocorreram 2,1 milhões de mortes e cerca de 2,5 milhões de novos casos ${ }^{1}$.

No Brasil, desde a identificação do primeiro paciente com Aids, em 1982, até junho de 2007, já foram identificados cerca de 474 mil casos da doença. Estima-se que aproximadamente 593 mil pessoas vivam atualmente com HIV ou Aids e, segundo parâmetros da OMS, a prevalência da infecção pelo HIV é de 0,61\% entre a população de 15 a 49 anos, sendo 0,42\% entre as mulheres e $0,80 \%$ entre os homens ${ }^{2}$.

Já nos primeiros casos ficou bem evidente o grave e progressivo comprometimento imunológico dos pacientes infectados pelo HIV, particularmente de sua imunidade celular. Tal fato acabava predispondo-os a neoplasias e infecções, a maioria de caráter oportunístico e estas em especial sempre trouxeram elevada morbimortalidade para os doentes de Aids, sendo elementos marcadores da síndrome.
Ao lado do sistema linfoide, o Sistema Nervoso Central (SNC) é um importante alvo para o HIV e o vírus tem sido frequentemente detectado no líquido cefalorraquidiano (LCR) e tecido cerebral desde o início da infecção e em toda a sua evolução, independentemente de apresentar sintomas neurológicos ${ }^{3}$. 0 vírus infecta e replica-se em macrófagos, micróglia e células multinucleadas da glia, mas está, principalmente, livre e presente no líquido cefalorraquidiano acelular $^{4}$

O SNC é o segundo local mais comum de manifestações clínicas. Isto pode ser explicado pelo fato de o vírus ser neurotrópico e o SNC um "santuário" para ele, além da pobre penetração das drogas antirretrovirais na presença de uma barreira hematoencefálica intacta 5 .

As manifestações neurológicas acometem 40\% a 70\% dos pacientes portadores do HIV no curso da sua infecção ${ }^{6,7}$, sendo que, em estudos de necropsia, a frequência pode chegar a mais de $90 \%{ }^{8}$. Cerca de $46 \%$ dos pacientes internados com Aids podem apresentar doença neurológica, seja como motivo principal da admissão hospitalar ou como intercorrências durante a internação ${ }^{9}$.

A natureza das alterações neurológicas é muito variada e qualquer parte do neuroeixo pode ser acometida. O determinante mais importante da susceptibilidade é o grau de imunossupressão. 0 diagnóstico diferencial é amplo e envolve etiologias infecciosas, neoplásicas, cérebro-vasculares, tóxico-metabólicas, nutricionais, 
autoimunes e relacionadas ao próprio vírus como neuropatias, mielopatias e alterações cognitivas. Também podem ocorrer associações de etiologias no mesmo paciente, o que é uma particularidade do imunodeprimido.

No curso da infecção pelo HIV o vírus entra no SNC podendo resultar em transtornos da função cognitiva causando déficits dos processos mentais, tais como atenção, aprendizado, memória, rapidez do processamento de informações, capacidade de resolução de problemas e sintomas sensoriais e motores.

Recentes avanços no tratamento da infecção pelo HIV aumentaram a expectativa de vida dos pacientes tornando mais provável que médicos e psicólogos encontrem na prática clínica diária pacientes com manifestações neuropsiquiátricas da doença.

As manifestações neurológicas mais comuns, ligadas diretamente ao HIV, são o transtorno cognitivo e motor menor e a demência associada ao HIV. No Brasil, as sequelas relacionadas às doenças oportunisticas do SNC, como neurotoxoplasmose, meningite tuberculosa e neurocriptococose, também são importantes causas de danos cognitivos e psiquiátricos. Portanto, o correto e precoce diagnóstico destas condições e a pronta intervenção terapêutica podem minimizar as complicações neuropsiquiátricas.

É de grande importância quantificar o número de pacientes com danos cognitivos, uma vez que afetam a qualidade de vida, função laborativa e aderência à terapia antirretroviral altamente ativa (HAART) ${ }^{10,11}$. Dano cognitivo é associado com aumento do risco de mortalidade, aumento do risco de desenvolver demência e a altas taxas de desemprego e isto ocorre mesmo na era HAART.

\section{Fisiopatologia e epidemiologia das alterações cognitivas}

O HIV atravessa a barreira hemato-encefálica por um mecanismo tipo cavalo de Tróia, usando macrófagos infectados ${ }^{12}$. No cérebro, o vírus infecta células gliais que em última instância secretam neurotoxinas que levam ao dano e morte neuronal ${ }^{13}$. A extensão deste dano é ligada ao nível do déficit neurológico clínico. Exames de necropsia de pacientes HIV positivos mostraram a presença de vírus em estruturas corticais e subcorticais, como lobos frontais, substância branca subcortical e núcleos da base ${ }^{14}$. Mecanismos que levam ao dano cognitivo ainda não são totalmente conhecidos, mas neurotoxinas liberadas pela micróglia e macrófagos periventriculares causam liberação de citocinas e quimiocinas, que levam à modificação da arquitetura sináptica do córtex. A apoptose ou morte celular programada é o mecanismo mais comum que leva à perda celular ${ }^{15}$

O HIV entra no Sistema Nervoso, invade suas células e produz lesões em todo tecido neural, derivando certos comprometimentos cognitivos ${ }^{16}$. É descrito perda neuronal sobre todo córtex frontal, atrofia cerebral e desmielinização, fundamentalmente nas zonas periventriculares, corpo caloso, cápsula interna, comissura anterior e tracto óptico ${ }^{17,18}$

O HIV pode permanecer latente no SNC por muitos anos e sua mera presença pode levar a déficits sutis no funcionamento cognitivo, entretanto estes déficits não são achados em todos pacientes, fato que levou alguns autores a proporem que ativadores periféricos possam estar envolvidos na fisiopatologia dos déficits. Pesquisas ainda são necessárias para determinar quais indivíduos ou subgrupos de pacientes são mais vulneráveis a complicações neurológicas ${ }^{15}$.

A demência associada à Aids é um efeito do próprio vírus em conjunto com a resposta do organismo infectado ${ }^{19}$. Pacientes com doença avançada apresentam-se com déficits em vários domínios cognitivos, enquanto pacientes com infecção pelo HIV, mas assintomáticos podem ter déficits sutis e limitados a poucos domínios cognitivos ${ }^{20}$. Geralmente estes pacientes HIV assintomáticos podem ter um dos dois padrões; depressão, lentidão psicomotora e diminuição da memória verbal ou diminuição do funcionamento cognitivo verbal e não-verbal na ausência de distúrbios do humor.

A demência pode ser manifestação inicial da Aids em 5\% dos $\operatorname{casos}^{21}$, mas geralmente aparece nos estágios avançados da infecção. A incidência anual, antes da HAART, era de 7\% a 14\% após o diagnóstico de Aids, sendo o risco cumulativo de desenvolver demência durante a vida de $5 \%$ a $20 \%{ }^{21,22}$.

Demência associada ao HIV contribui para morbidade da infecção e é um fator de risco para mortalidade ${ }^{23}$. Antes do uso da HAART acima de $15 \%$ dos indivíduos com Aids tinham demência e acima de $15 \%$ transtorno cognitivo motor menor ${ }^{24}$. Infecção avançada é um fator de risco para o desenvolvimento de demência tanto na era pré quanto na pós HAART ${ }^{25}$

Houve um declínio nos relatos da incidência de demência com a introdução da HAART e consequente reconstituição imune nos países desenvolvidos ${ }^{25,26}$, mas deve-se ter cautela na interpretação destes dados que subestimam o impacto atual desta doença ${ }^{27}$. Há simultaneamente relatos de aumento da prevalência e um aumento da incidência de déficit cognitivo menor comparado à demência. Aliado a isto houve um aumento da proporção de indivíduos com alterações cognitivas diagnosticados com uma melhor imunidade (contagem de linfócitos T CD4 maior que 200 céls $/ \mathrm{mm}^{3}$ ) e também um aumento da incidência da demência como doença definidora de Aids ${ }^{26,27}$. Encefalopatia pelo HIV continua presente em cerca $25 \%$ dos pacientes que submetem à autópsia. Esta taxa não foi alterada pela HAART ${ }^{28}$. Ao contrário, Neuenburg et al. (2002) ${ }^{29}$ encontraram aumento da incidência de encefalopatia pelo HIV com o passar do tempo.

Analisados tais dados verifica-se que a demência pelo HIV continua a ser importante fonte de morbidade na infecção pelo HIV ${ }^{27}$. A HAART prolonga a vida e restaura a resposta imune para patógenos não-HIV, mas não previne a patologia direta relacionada ao HIV no cérebro, apesar de diminuir a gravidade dos achados anátomo-patológicos em autópsias ${ }^{29}$. Nos últimos anos ocorreu diminuição da incidência de doenças neurológicas, tanto as secundárias (oportunísticas), quanto as primárias (demência ligada ao HIV). No entanto, a demência associada ao HIV continua a ser a causa mais comum de demência em jovens com idade inferior a 40 anos ${ }^{25,29-31}$

Com a HAART, a incidência da demência associada ao HIV vem diminuindo 26,29,32, mas a prevalência aumentando devido a maior sobrevida dos pacientes infectados pelo HIV e o aparecimento de formas mais leves de dano cognitivo ${ }^{33}$.

A diminuição da morbidade e mortalidade com a HAART leva a um aumento do número de pessoas vivendo com Aids, podendo representar um grupo "neurologicamente vulnerável" para doença neurológica, com o SNC servindo como um santuário para replicação do HIV parcialmente suprimido ${ }^{34}$.

A sobrevida média entre o diagnóstico da demência associada ao HIV e o óbito na era pré-HAART era de seis meses e na era pós HAART é de 44 meses $^{35}$. A média de linfócitos T CD4 por ocasião do diagnóstico era 50 a 100 céls $/ \mathrm{mm}^{3}$ e agora é 160 céls $/ \mathrm{mm}^{3} 36$. 
A razão para a elevação da média de linfócitos T CD4 por ocasião do diagnóstico de demência não é clara, mas pode relacionar-se à falha da restauração de um defeito específico da função imune relacionada à demência pelo HIV ou pode ainda significar que a duração da infecção pelo HIV é um fator importante no desenvolvimento da demência ou mesmo a combinação de ambos os fatores ${ }^{37}$.

Déficits cognitivos desenvolviam em $30 \%$ das pessoas vivendo com Aids e 15\% tinham franca demência na era pré-HAART ${ }^{38-40}$. $\mathrm{Na}$ era HAART, alterações cognitivas afetam até $20 \%$ a $30 \%$ dos indivíduos HIV 25

\section{Diagnóstico das alterações cognitivas da AIDS}

Os termos Complexo Demência-Aids, Demência-HIV, encefalopatia pelo HIV e complexo demência associado-HIV ou Aids são sinônimos. Graus menores de dano cognitivo, motor e funcional, na qual não são suficientes para o diagnóstico de demência é chamado transtorno cognitivo-motor menor associado-HIV e nem sempre pacientes com estes transtornos evoluem para franca demência ${ }^{5}$.

O termo Complexo Demência associado ao HIV refere-se à constelação de sintomas e sinais cognitivos, motores e comportamentais e é classificado como uma demência "subcortical" conforme estudos de neuroimagem e anátomo-patológicos, portanto tem alguma similaridade com a demência das doenças de Huntington e Parkinson (Quadro 1).

A característica essencial do Complexo Demência associado ao HIV-1 é o seu comprometimento cognitivo que pode ser acompanhado de disfunção motora, comportamental ou ambas. No entanto, alguns pacientes com alterações cognitivas podem não ter alterações comportamentais e outros podem não ter alterações motoras.

\section{Quadro 1 - Sinais e sintomas da demência pelo HIV}

Cognitivo Perda de memória visuoespacial (ex. objetos em lugares trocados), perda da coordenação visuomotora, esquecimentos, dificuldade de concentração e atenção, lentidão no pensamento (compreensão e processamento), dano na memória verbal (ex. dificuldade de achar palavras)

Tardios: desorientação temporal e espacial, mutismo

Motor Marcha instável, perda do balanço, lentidão dos movimentos, fraqueza MMII*, declínio das habilidades motoras finas, piora da escrita, incoordenação Estágio inicial: lentidão de movimentos rápidos, tremor ocasional, marcha com pequenos passos Estagio tardio: hiperreflexia, sinal de Babinski, pode associar polineuropatia

Estágio terminal: tetrapelgia espástica, incontinência urinária e fecal

Emocional Perda da iniciativa (apatia), irritabilidade, mania, psicose de início recente

Comporta- Retardo psicomotor (ex. lentidão na fala ou no tempo mental de resposta), alterações de personalidade, afastamento de atividades sociais
O início da demência pelo HIV é insidioso e em seus estágios iniciais o paciente pode queixar de dificuldade de concentração, apatia e lentidão mental. Estes sintomas podem ser confundidos com depressão. Nos estágios mais tardios a síndrome progride aparecendo alterações mais específicas de perda de memória, dificuldade de leitura e alterações da personalidade associadas à lentidão motora ${ }^{14}$.

Odesenvolvimento da demência ocorretipicamenteem semanas e meses, sendo que a ocorrência de sintomatologia aguda aponta para outra etiologia. Alguns pacientes podem mostrar estabilidade do quadro por vários meses ou anos com progressão muito lenta. O quadro pode permanecer estático ou flutuar. Pode melhorar com a HAART e piorar na presença de graves distúrbios metabólicos.

O exame do paciente pode revelar bradpsiquismo, alterações da motilidade ocular, diminuição da expressão facial, hipofonia, dano na coordenação e equilíbrio, tremor e sinais de liberação frontal. Os estágios finais são caracterizados por quase mutismo ${ }^{14}$, no entanto atualmente, devido ao uso da HAART raramente são vistos.

Sintomas psiquiátricos como agitação, mania, alucinações e paranoia podem também ocorrer nos estágios tardios ${ }^{41}$. Sinais como rigidez de nuca e déficits focais como hemiparesia e afasia não são comuns na demência pelo HIV, bem como a presença de crises epilépticas focais e generalizadas o que deve alertar para outros diagnósticos. Amnésia e agnosia também são incomuns até os estágios terminais da doença.

O diagnóstico é baseado na história clínica, exame neurológico e cognitivo, além de exclusão de outras causas através de métodos de imagem e LCR ${ }^{42}$. A ressonância nuclear magnética de encéfalo é superior à tomografia computadorizada de crânio e pode demonstrar lesões hiperintensas e relativamente simétricas na substância branca. Também pode ocorrer hipotrofia cortical e hidrocefalia, no entanto nenhum destes sinais é específico de demência pelo HIV e a doença pode estar presente mesmo com um exame normal. Pacientes com transtorno cognitivo/motor menor associado ao HIV-1 tem geralmente exames de imagens normais.

Dano cognitivo associado com infecção do HIV é dividido em duas entidades distintas segundo a Academia Americana de Neurologia: transtorno cognitivo motor menor e demência associada ao HIV (Quadro 2) ${ }^{43}$. Critérios diagnósticos baseiam-se em um déficit adquirido combinado de habilidades cognitivas incluindo funções motoras (como bradicinesia, alteração da marcha e hipertonia), comportamentais (como apatia, irritabilidade e labilidade emocional) e cognitivas (como atenção, concentração, memória, processamento de informação e linguagem). A diferença entre as duas entidades é o grau de comprometimento das atividades da vida diária. A característica essencial da demência é a perda das funções intelectuais em gravidade suficiente para interferir com o funcionamento social e ocupacional ${ }^{43}$.

Nos estágios menos avançados é necessário detalhar a avaliação neuropsicológica para determinar o grau e natureza do dano cognitivo e identificar morbidades tais como depressão e ansiedade ${ }^{44,45}$.

Com a HAART houve alterações na história natural e no curso dos sintomas cognitivos como o aumento dos níveis médios de linfócitos T CD4 nos pacientes com demência, aumento da sobrevida e aparecimento de formas mais leves ${ }^{37}$. Portanto é importante reconhecer precocemente danos sutis, já que, em alguns casos, podem significativamente melhorar a chance de reversão da demência com HAART $^{5}$, melhorar a qualidade de vida com tratamentos adicionais 


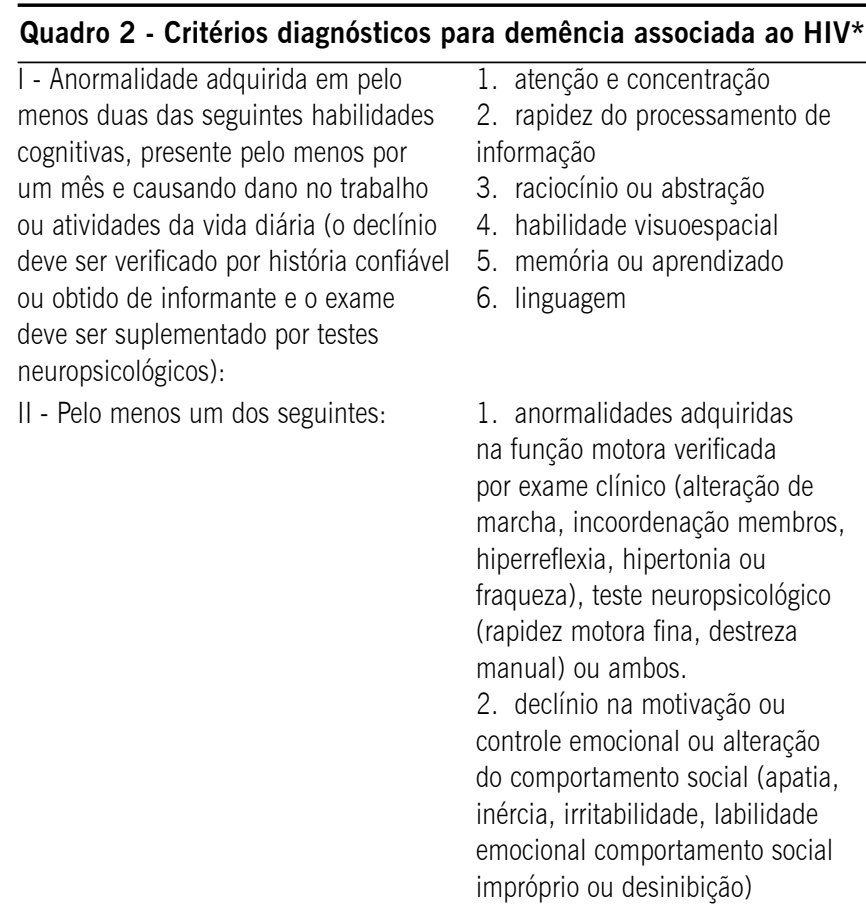

III - Ausência de alteração do nível e estado de consciência durante período suficiente para estabelecer a presença de I

IV - Ausência de outras causas de anormalidades cognitivas, motoras ou comportamental (ex. infecções ou malignidades oportunísticas ativas do SNC**, distúrbios psiquiátricos, abuso de substância).

* Modificado da referência 43: Working Group of the American Academy of neurology AIDS Task Force.

** SNC; sistema nervoso central

${ }^{46}$ e mesmo possibilitar a monitorização da aderência do paciente à medicação. Além do fato de poder melhorar a orientação para os familiares sobre as alterações comportamentais e cognitivas do paciente.

Fatores de risco para desenvolver demência são o diagnóstico da Aids em idade avançada, elevada carga viral plasmática e no líquido cefalorraquidiano, contagem de linfócitos T CD4 menor que 100 céls $/ \mathrm{mm}^{3}$, baixa concentração de hemoglobina e a progressão da doença sistêmica ${ }^{5}$.

Os diagnósticos de demência e de distúrbio cognitivo menor são de exclusão, ou seja, patógenos infecciosos, tumores e causas metabólicas de encefalopatia devem ser investigados antes de o déficit cognitivo ser atribuído a infecção pelo HIV.

A avaliação neuropsicológica tem um papel fundamental na identificação e diagnóstico de transtornos cognitivos associados ao HIV e é usada para quantificar alterações em processos cognitivos associado com o tratamento.
Testes neuropsicológicos são sensíveis para detectar transtornos cognitivos na infecção pelo HIV-1 e devem incluir os seguintes domínios: atenção/concentração; rapidez do processamento da informação; função executiva; raciocínio/abstração; memória/ aprendizado; habilidade visuoespacial; e funcionamento motor.

$\mathrm{Na}$ análise destes testes devem ser considerados possíveis fatores de confusão ou associados que podem alterá-los como uso de álcool, drogas ilícitas e certas drogas terapêuticas, antecedentes de doenças neurológicas (ex. trauma craniano) ou psiquiátricas (ex. depressão maior ou transtornos de aprendizagem). Os testes neuropsicológicos são bastante úteis, mas sozinhos não são capazes de determinar a presença do Complexo Demência associado ao HIV-1 ou transtorno cognitivo/motor menor.

Testes rápidos de avaliação cognitiva como o mini exame do estado mental, que é útil para demências "corticais" como na demência de Alzheimer, não mostrou ser útil para demências "subcorticais" como a associada ao HIV $31,47$.

Em 1995, Power et al. ${ }^{48}$ descreveram um teste específico para triagem de dano cognitivo em pacientes com Aids, porém foi testado em pacientes americanos e com nível social, cultural e econômico diferente da população brasileira. Esta escala de demência pelo HIV inclui testes que avaliam rapidez motora (tempo gasto para escrever o alfabeto), memória (lembrança de quatro palavras após cinco minutos), praxia construcional (tempo para copiar um cubo) e função executiva (número de erros de testes de movimentos oculares antisacádicos). Este último subteste, de erros de movimentos antissacádicos, foi demonstrado ser difícil de ser aplicado pelo não neurologista ${ }^{49}$. Os subtestes de cópia do cubo e escrita do alfabeto podem ser difíceis para indivíduos com baixo nível sociocultural.

Sackor N. et al. (2005) ${ }^{31}$ descreveram uma escala (International HIV Dementia Scale), que foi validada para triagem em uma população com baixa escolaridade (Uganda-África), mas esta escala apresentava escores entre dementes e não-dementes muito próximos e foi aplicada em uma amostra pequena de pacientes. Nenhuma destas escalas foi validada para o Brasil e não sabemos sua real sensibilidade e especificidade.

Recentemente foi revista a nosologia dos transtornos neurocognitivos associados ao HIV ${ }^{50}$. Estes critérios modificados são baseados nos critérios da Academia Americana de Neurologia (1991) ${ }^{43}$ e em pesquisas e observações feitas por publicações após a introdução da HAART. Foram reconhecidas três condições: transtornos neurocognitivo assintomático, transtorno neurocognitivo leve associado ao HIV e demência associada ao HIV (Quadro 3).

A mais importante alteração foi a introdução da categoria transtorno neurocognitivo assintomático baseado na observação que alguns indivíduos têm demonstráveis transtornos cognitivos nos testes neuropsicológicos sem qualquer anormalidade no funcionamento da vida diária. O critério da AAN não reconhece este subgrupo de pacientes que pode chegar a $15 \%$ dos pacientes infectados pelo HIV.

Outro fato interessante observado após a HAART é que cerca de $20 \%$ dos pacientes podem ter alterações cognitivas bidirecionais, ou seja, pode flutuar de normais para anormais, nos seus diferentes níveis de gravidade, e vice-versa.

Os testes neuropsicológicos empregados para classificação dos três transtornos cognitivos necessitam informar quanto à presença e gravidade do transtorno cognitivo, à presença e gravidade do declínio funcional e o grau na qual o transtorno cognitivo 


\section{Quadro 3 - Critérios revisados para transtornos neurocognitivos associado ao HIV}

\section{Transtorno neurocognitivo assintomático associado ao HIV}

1-Transtorno adquirido no funcionamento cognitivo envolvendo pelo menos dois domínios documentado por pelo menos um DP abaixo da média de testes neuropsicológicos estandardizados e apropriados para idade e educação. A avaliação deve incluir as funções; visual/linguagem, atenção/ memória de trabalho, abstração/função executiva, memória, rapidez do processamento de informação, habilidades motoras e percepção-sensorial.

\section{2-0 transtorno cognitivo não interfere com as atividades da vida diária}

\section{3-0 transtorno cognitivo não encontra critérios para delirium ou demência}

4- Não existe evidência de outra causa pré-existente para do déficit

\section{Transtorno neurocognitivo leve associado ao HIV}

1-Transtorno adquirido no funcionamento cognitivo envolvendo pelo menos dois domínios documentado por pelo menos um DP abaixo da média de testes neuropsicológicos estandardizados e apropriados para idade e educação. A avaliação deve incluir as funções; visual/linguagem, atenção/ memória de trabalho, abstração/função executiva, memória, rapidez do processamento de informação, habilidades motoras e percepção-sensorial

2- 0 transtorno cognitivo leva a pelo menos leve interferência nas atividades da vida diária (pelo menos um dos seguintes):
a) Redução da acuidade mental, ineficiência no trabalho, nas
b) Observação por terceiros que 0 indivíduo tem leve declínio na acuidade atividades do lar ou no funciona- mento social autorreportado pelo paciente
mental com resultante ineficiência no
trabalho, atividades do lar e funciona- mento social

3- 0 transtorno cognitivo não encontra critérios para delirium ou demência

4- Não existe evidência de outra causa preexistente para o déficit

\section{Demência associada ao HIV}

1- Marcado déficit adquirido do funcionamento cognitivo envolvendo pelo menos dois domínios; tipicamente o distúrbio é em múltiplos domínios, especialmente no aprendizado de novas informações, lentidão no processamento de informação e déficit atenção/concentração. 0 dano cognitivo deve ser verificado por teste neuropsicológico com pelo menos dois DP abaixo da média de testes neuropsicológicos estandardizados e apropriados para idade e educação em dois ou mais domínios.

2- 0 déficit cognitivo produz marcada interferência nas atividades da vida diária (trabalho, atividades em casa e vida social)

\section{3- 0 transtorno cognitivo não encontra critérios para delirium}

4- Não existe evidência de outras causas pré-existentes para do déficit (ex. infecções do SNC, AVC, doença neurológica pré-existente, uso de drogas ilícitas, etc.)

Fonte; Modificado de Antinori A., et al. 2007. e funcional são influenciados por condições co-mórbidas presente como doenças oportunisticas do SNC, uso de drogas e distúrbios psiquiátricos.

\section{Perfil de alterações cognitivas e comprometimento funcional secundários à infecção pelo HIV}

As habilidades para executar tarefas da vida diária é um complemento importante na avaliação cognitiva. Sua redução pode representar um indicativo de demência.

O comprometimento cognitivo é clinicamente significativo quando afeta o funcionamento das atividades diárias. Os indivíduos infectados com alterações cognitivas leves podem apresentar problemas ocupacionais, mesmo nos estágios iniciais da doença. Indivíduos HIV-positivos com alterações cognitivas, principalmente, em funções executivas e processos de aprendizagem apresentam, geralmente, menor desempenho no trabalho.

Os problemas de atenção, memória de trabalho, abstração/ função executiva são os domínios cognitivos mais diretamente relacionados às dificuldades do funcionamento das atividades instrumentais diárias nos pacientes com infecção pelo HIV. Por outro lado, o quadro depressivo pode causar alteração na capacidade do paciente de gerenciar suas atividades diárias devido ao rebaixamento do estado de ânimo. Danos nestes domínios cognitivos podem levar os pacientes portadores do HIV a apresentar dificuldades significativas na administração da medicação, bem como, incluir o tratamento com antirretrovirais como parte das suas atividades diárias ${ }^{51,52}$.

As medidas de avaliação funcional são utilizadas para investigar os dados de auto manutenção (vestir-se, higiene e alimentação) e também as atividades diárias como capacidade de fazer compras, cozinhar, lavar, administrar a medicação e gerenciar a vida financeira.

\section{Suporte Financeiro:}

CNPq (bolsa de pós-doutorado)

\section{Conflito de interesse: não há}

\section{SUMmARY}

Cognitive alterations associated With HIV-1 infection and Aids

Primary neurological complications of AIDS include cognitive deficits such as HIV-associated dementia and milder forms such as cognitive/motor disorders, which cause changes in daily activities and reduce the quality of life of patients. Infection with HIV-1 is the most common, predictable and treatable cause of cognitive deficits in individuals with less than 50 years of age. . Despite advances in the understanding of clinical characteristics, pathogenesis and neurobiological aspects and widespread use of highly active antiretroviral therapy (HAART), neurological complications and cognitive deficits still persist with serious personal and socioeconomic consequences, thus representing a great therapeutic challenge. In the pre-HAART era dementia was a common complication of infection whose incidence declined during the HAART era. However, prevalence of dementia has increased, especially that of milder forms due to the increased number of infected individuals and increased life expectancy. Cognitive alterations associated with HIV are typically sub cortical and can be associated with behavioral 
and motor disorders. These syndromes are clinically diagnosed by neuropsychological tests, while neuroimaging and analysis of cerebrospinal fluid contribute to diagnosis. This review is an update on current epidemiological status, clinical characteristics and diagnosis of cognitive complications observed during the course of HIV infection. [Rev Assoc Med Bras 2010; 56(2): 242-7]

KEY WORDS: AIDS dementia complex. HIV. Acquired immunodeficiency syndrome. Activities of daily life. Review.

\section{REFERÊNCIAS}

1. World Health Organization. Aids epidemic update 2007. Geneva: Joint United Nations Program on HIV/AIDS; 2007.

2. Ministério da Saúde. Dados de AIDS. [citado 20 fev 2009]. Disponível em: http:// www.aids.gov.br.

3. Christo PP, Greco DB, Aleixo AW, Livramento JA. HIV-1 RNA levels in cerebrospinal fluid and plasma and their correlation with opportunistic neurological disease in a Brazilian AIDS reference Hospital. Arq Neuropsiquiatr. 2005;63:907-913.

4. Spector SA, Hsia K, Pratt D, Lathey J, McCutchan JA, Alcaraz JE, et al. The HIV Neurobehavioral Research Center Group. Virologic markers of human immunodeficiency virus type 1 in cerebrospinal fluid. J Infect Dis. 1993;168:68-74.

5. McArthur JC, Haughey N, Gartner S, Conant K, Pardo C, Nath A, et al. Human immunodeficiency virus-associated dementia: an evolving disease. J Neurovirol. 2003;9:205-21.

6. Berger JR, Moskowitz L, Fischl M, Kelley RE. Neurologic disease as the presenting manifestation of acquired immunodeficiency syndrome. South Med J. 1987;80:683-6.

7. Levy RM, Bredesen DE, Rosenblum LV. Neurological manifestation of acquired immune deficiency syndrome (AIDS): experience at UCSF and review of the literature. J Neurosurg. 1985;62:475-95.

8. Chimelli L, Rosemberg S, Hahn MD, Lopes MB, Netto MB. Pathology of the central nervous system in patients infected with the human immunodeficiency virus (HIV): a report of 252 autopsy cases from Brazil. Neuropathol Appl Neurobiol. 1992;18:478-88.

9. Oliveira JF, Greco DB, Oliveira GC, Christo PP, Guimarães MDC, Oliveira RC Neurological disease in HIV-1-infected patients in the era of highly active antiretroviral treatment: a Brazilian experience. Rev Soc Bras Med Trop. 2006;39:146-5.

10. McArthur JC. HIV dementia: an evolving disease. J Neuroimmunol. 2004; 157(1-2):3-10.

11. Price RW, Yiannoutsos CT, Clifford DB, Zaborski L, Tselis A, Sidtis JJ, et al. Neurological outcomes in late HIV infection: adverse impact of neurological impairment on survival and protective effect of antiviral therapy. AIDS 1999;13:1677-1685.

12. Lawrence DM, Major EO. HIV-1 and the brain: connections between HIV-1associated dementia, neuropathology and neuroimmunology. Microbes Infect. 2002,4:301-8.

13. Clifford DB. AIDS dementia. Med Clin North Am. 2002;86:537-50.

14. Navia BA, Jordan BD, Price RW. The AIDS dementia complex I: clinical features. Ann Neurol.1986;19:517-24.

15. Dubé B, Benton T, Cruess DE, Evans DL. Neuropsychiatric manifestations of HIV infection and AIDS. J Psychiatry Neurosci. 2005;30:237-46.

16. Brew BJ, Rosenblum M, Price RW. AIDS dementia complex and primary HIV brain infection. J Neuroimmunol. 1988;20:133-40.

17. Everall IP, Luthert PJ, Lantos PL. Neuronal loss in the frontal cortex in HIV infection. Lancet. 1991;337(8750):1119-21.

18. Bell JE An update on the neuropathology of HIV in the HAART era. Histopathology. 2004;45:549-59.

19. Atkinson JH, Grant I. Natural history of neuropsychiatric manifestations of HIV disease. Psychiatr Clin North Am. 1994; 17(1):17-33.

20. Heaton RK, Grant I, Butters N, White DA, Kirson D, Atkinson JH, et al. The HNRC 500-neuropsychology of HIV infection at different disease stages. J Int Neuropsychol Soc. 1995;1:231-51

21. McArthur JC, Hoover DR, Bacellar H, Miller EN, Cohen BA, Becker JT, et al. Dementia in AIDS patients: incidence and risk factors. Multicenter AIDS Cohort Study. Neurology. 1993;43:2245-52.

22. Day JJ, Grant I, Atkinson JH, Brysk LT, McCutchan JA, Hesselink JR, et al. Incidence of AIDS dementia in a two-year follow-up of AIDS and ARC patients on na initial phase II AZT placebo-controlled study: San Diego cohort. J Neuropsychiatry Clin Neurosci. 1992:4:15-20.

23. Sacktor NC, Bacellar H, Hoover DR, Nance-Sproson TE, Selnes OA, Miller EN, et al. Psychomotor slowing in HIV infection: a predictor of dementia, AIDS and death. J Neurovirol. 1996;2:404-10.

24. McArthur JC, Sackor N, Selnes O. Human immunodeficiency vírus-associated dementia. Semin Neurol. 1999;19:129-50.

25. Sackor N. The epidemiology of human immunodeficiency virus-associated neurological disease in the era of highly active antiretroviral therapy. J Neurovirol. 2002;2(Suppl):115-21.
26. Sacktor N, Lyles RH, Skolasky R, Kleeberger C, Selnes OA, Miller EN, et al. Multicenter AIDS Cohort Study. HIV-associated neurologic disease incidence changes: Multicenter AIDS Cohort Study, 1990-1998. Neurology 2001;56:257-60.

27. Valcour VG, Shikuma CM, Watters MR, Sackor NC. Cognitive impairment in older HIV-1-seropositive individuals: prevalence and potential mechanisms. AIDS 2004;18(Suppl 1):S79-S86

28. Masliah E, DeTeraza RM, Mallory ME, Hansen LA. Changes in pathological findings at autopsy in AIDS cases for the last 15 years. AIDS. 2000;14:69-74.

29. Neuenburg JK, Brodt HR, Herndier BG, Bickel M, Bacchetti P, Price RW, et al. HIV-1 related neuropathology, 1985 to 1999: rising prevalence of HIV encephalopathy in the era of highly active antiretroviral therapy. J Acquir Immune Defic Syndr. 2002;31:171-7.

30. Arminio Monforte A, Cinque P, Mocroft A, Goebel FD, Antunes F, Katlama C, et al. EuroSIDA Study Group. Changing incidence of central nervous system disease in the EuroSIDA cohort. Ann Neurol. 2004:55:320-8.

31. Sacktor NC, Wong M, Nakasujja N, Skolasky RL, Selnes OA, Musisi S, et al. The International HIV Scale: a new rapid screening test for HIV dementia. AIDS 2005; 19:1367-74

32. Dilley JW, Schwarcz S, Loeb L, Hsu LNelson K, Scheer S. The decline of incidence cases of HIV-associated neurological disorders in San Francisco,1991-2003. AIDS. 2005; 19:634-5.

33. Cysique LA, Maruff P, Brew BJ. Prevalence and pattern of neuropsychological impairment in human immunodeficiency virus-infected/acquired immunodeficiency syndrome (HIV/AIDS) patients across pre and pos-highly active antiretroviral therapy eras: a combined study of two cohorts. J Neurovirol. 2004;10:350-7.

34. Pialoux G, Fournier S, Moulignier AA, Poveda J-D, Clavel F, Dupont B. Central nervous system as a sanctuary for HIV-1 infection despite treatment with zidovudine, lamivudine and indinavir [letter]. AIDS. 1997;11:1302-3.

35. McArthur JC, Brew JB, Nath A. Neurological complications of HIV infection. Lancet Neurol. 2005;4:543-55.

36. Dore G, Correll P, Kaldor J, Brew BJ. Changes to the natural history of AIDS dementia complex in the era of HAART. AIDS. 1999;13:1249-53.

37. Brew BJ. Evidence for a change in AIDS dementia complex in the era of highly active antiretroviral therapy and the possibility of new forms of AIDS dementia complex. AIDS; 2004:18(Suppl1); S75-S78.

38. Janssen ES, Wanyanwi OC, SeliK RM, Stehr-Green JK. Epidemiology of human immunodeficiency encephalopathy in the United States. Neurology. 1992;42:1472-6.

39. McArthur JC, McClernon DR, Cronin MF. Relationship between human immunodeficiency virus-associated dementia and viral in cerebrospinal fluid and brain. Ann Neurol. 1997;42:689-98

40. Navia BA, Price RW. The acquired immunodeficiency syndrome dementia complex as presenting or sole manifestation of human immunodeficiency virus infection. Arch Neurol. 1987;44:65-69.

41. Perry SW. Organic mental disorders caused by HIV: update on early diagnosis and treatment. Am J Psychiatry. 1990;147:696-710.

42. Diesing TS, Swindells S, Gelbard H, Gendelmam HE. HIV-1-Associated dementia: a basic science and clinical perspective. AIDS Read. 2002;12:358-68.

43. Working Group of America Academy of Neurology AIDS Task Force. Nomenclature and research case definitions for neurologic manifestations of human immunodeficiency virus-type 1(HIV-1) infection. Neurology. 1991;41:778-85.

44. Berger JR, Brew B. An international screening tool for HIV dementia. AIDS. 2005; 19:2165-6

45. Price RW, Brew BJ. The AIDS dementia complex. J InfectDis. 1988;158:1079-83.

46. Tozzi V, Balestra P, Galgani S, Murri R, Bellagamba R, Narciso P, et al. Neurocognitive performance and quality of life in patients with HIV infection. AIDS Res Hum Retroviruses. 2003;19:643-53.

47. Goodkin K. Evolution of neuro-AIDS During the HAART Era. J Neuro-AIDS. 2002;21-17.

48. Power C, Selnes OA, Grim JA, McArthur JC. HIV Dementia Scale: a rapid screening test. J Acquir Immune Defic Syndr Hum Retrovirol. 1995;8:273-8.

49. Davis HF, Skolasky RL Jr, Selnes OA, Burgess DM, McArthur JC. Assessing HIVassociated dementia: modified HIV dementia scale versus the Grooved Pegboard. AIDS Read. 2002;12:29-31.

50. Antinori A, Arendt G, Becker JT, Brew BJ, Byrd DA, Cherner M, et al. Updated research nosology for HIV-associated neurocognitive disorders. Neurology 2007; 69(18): 1789-99.

51. Hinkin CH, Hardy DJ, Mason KI, Castellon SA, Durvasula RS, Lam MN et al. Medication adherence in HIV-infected adults: effect of patient age, cognitive status, and substance abuse. AIDS. 2004;18(Suppl. 1):S19-S25.

52. Christo PP. Aspectos neuropsiquiátricos e neuropsicológicos da infecção pelo HIV e da AIDS. In: Fuentes D, Leandro F Malloy-Diniz LF, Pires Camargo CH, Moreira Cosenza R, organizadores. Neuropsicologia terórica e prática. Porto Alegre/RS: Artmed; 2008. p.335-55.

Artigo recebido: 01/06/09

Aceito para publicação: 8/11/09 